\title{
A review of computerized tomography with application to two-phase flows
}

\author{
P MUNSHI
}

Nuclear and Mechanical Engineering, Indian Institute of Technology, Kanpur 208 016, India

MS received 27 November 1989; revised 11 May 1990

\begin{abstract}
The technique of computerized tomography (CT) has established itself as a leading tool in diagnostic radiology over the past twenty years and is catching on fast in the non-destructive evaluation area in a variety of situations.

CT instrumentation involves a source and a detector system to scan the object of interest. The source can be acoustic, microwave, X-ray, gamma-ray, etc. depending upon the type of material being investigated. For fluid-flows, gamma-rays are quite suitable. There are basically two types of data collection geometries - fan-beam and parallel beam. Fan-beam requires less number of sources as one source feeds several detectors arranged in a fan-beam.

The use of $\mathrm{CT}$ in multi-phase flow studies has been limited presently to only a few laboratory experiments and the results indicate that $\mathrm{CT}$ does hold a lot of promise as an effective investigative methodology to understand some of the complex phenomena encountered in multi-phase flows. Some new CT algorithms developed specifically for pipe-flows have shown good results on some air-water flow data for a $15 \mathrm{~cm}$ dia pipe.
\end{abstract}

Keywords. Tomography; image reconstruction; two-phase flow; voidfraction.

\section{Introduction}

The area of medical imaging has been revolutionized with the introduction of X-ray based scanning machines involving the concept of computerized tomography (CT). The first Cr scanner was developed by Hounsfield (1973) who was later on awarded the Nobel prize for medicine. These CT machines have been in the market for the past 17 years and have proved to be excellent tools in the diagnosis of cancerous tissues at a very early stage. The power of $\mathrm{Cr}$ was quickly recognised by the researchers in the non-destructive testing (NDT) area and several machines have been developed

A list of symbols used in given at the end of the paper 
specifically for NDT work, e.g., Miyoshi et al (1987) and McClellan \& Tow (1986). The engineering and physics of $\mathrm{CT}$ is discussed in great detail in the work of Herman (1980) while the mathematics of CT has been elaborately described by Natterer (1986). The book by Deans (1983) lists several uses of CT other than the two applications mentioned above.

Tomographic scanners incorporating ultrasonics have also been developed but are not discussed here. This paper deals specifically with radiation based $\mathrm{CT}$ which generally involves X-ray or gamma-ray sources and detectors.

\subsection{Historical perspective}

Radon (1917) proved that any arbitrary function could be recovered from its set of line integrals taken along various chords and various directions. However, the method of Radon could not be implemented due to inherent mathematical complexities. Bracewell (1956) reported an application of $\mathrm{CT}$ in radio astronomy and Cormack (1963) derived an inversion formula, both of which were closer to being implementable as compared to Radon's solution. Bracewell \& Riddle (1967) and Ramachandran \& Lakshminarayan (1970) showed how computations involved in the CT methodology could be accelerated by adopting the use of convolutions. However, it was almost 50 years after the publication of Radon's paper that a CT machine was pioneered by Hounsfield (1973).

\subsection{Preliminaries}

Single beam mono-energetic radiation attenuation phenomenon in a plane can be represented by

$$
N=N_{0} \exp \left[-\int_{c} \mu(r, \phi) \mathrm{d} l\right] .
$$

The value of $\mu$ is characteristic of a material for a given type of radiation and the energy of radiation. Equation (1) considers $\mu$ to be a two-dimensional function of position, as the path of radiation is assumed to be restricted to a plane. The energy dependence of $\mu$ is also ignored. This simplifying assumption is valid for monoenergetic radiation sources, e.g. $\boldsymbol{\gamma}$-rays, and suitable corrections are incorporated to handle X-ray sources which have a distributed energy spectrum. We note that when the cross-section of interest has a uniform distribution of the absorbing material then (1) reduces to,

$$
N=N_{0} \exp (-\mu \Delta l),
$$

where $\mu$ is no longer a function of position.

In general, any cross-section of interest will have non-uniform distribution. Rewriting (1) as,

where

$$
p=\int_{c} \mu(r, \phi) \mathrm{d} l,
$$

$$
p=\ln \left(N_{0} / N\right)
$$

We observe that recovering $\mu$ from the given data $p$ is not a straightforward 
proposition. Radon (1917) showed that it is possible to recover $\mu$ from a set of several $p$-values measured along various chords, $c$. These $\mu$-values can then be suitably calibrated to give the density values if so desired by the user.

\section{Data collection modes}

The CT methodology requires attenuation data [given by (3)] collected by an array of radiation detectors for the reconstruction of the function $\mu(r, \phi)$. Two popular data collection modes are termed as parallel-beam geometry (PBG) and fan-beam geometry (FBG).

\subsection{Parallel-beam geometry}

CT scanners based on PBG mode have several pairs of radiation source and radiation detector systems which scan the object completely. Figure 1 depicts the configuration for a generic PBG scanner. The source-detector pairs are spaced uniformly and the object to be imaged is stationed on a table which can be rotated to give different values of $\theta$. The line SD represents the path of the data ray. The perpendicular distance of the ray from the centre of the object, which happens to be the origin of the frame of reference chosen for derivational and computational convenience, is denoted by $s$. Several SD pairs collect the data $p$ for a given $\theta$. This set of $p$ is known as 'projection'. The object table is rotated to get several sets of $p$ for different values of $\theta$, i.e., different 'views'. The data thus collected is denoted by $p(s ; \theta)$, the semi-colon indicating the procedure of data collection.

We note that if the object has to be kept stationary, then the SD system has to be rotated to collect the data for various 'views'.

\subsection{Fan-beam geometry}

The medical scanners incorporate the FBG collection mechanism which involves a rotating module including a source and an array of detectors stationed in an arc around the patient. Figure 2 shows the FBG configuration. To distinguish fan-beam

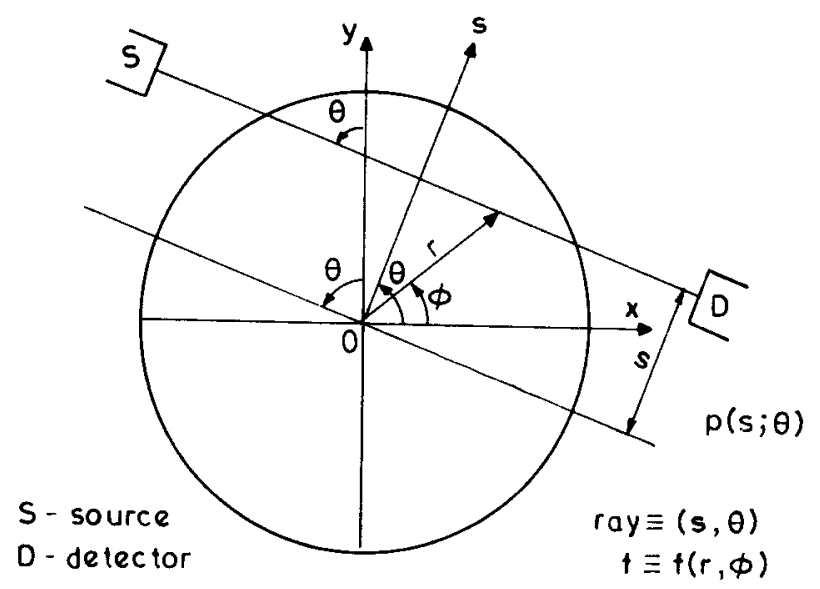

Figure 1. Parallel-beam geometry. 


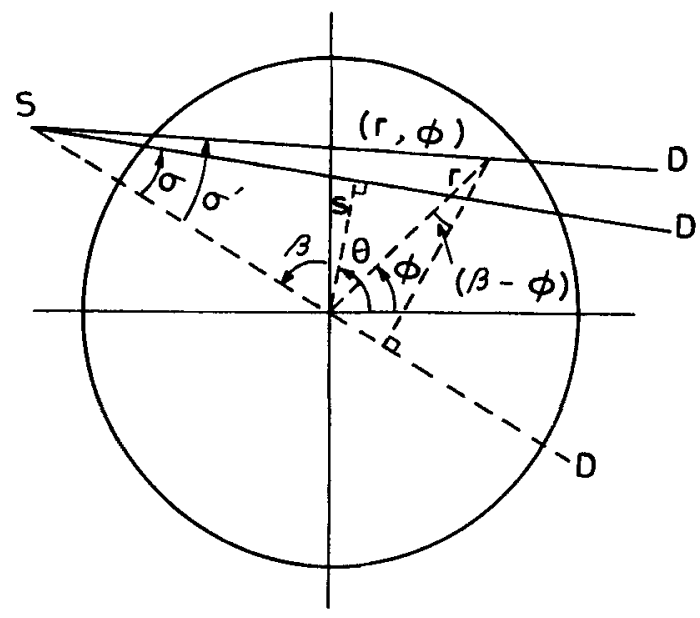

Figure 2. Fan-beam geometry.

from parallel-beam data, the FBG data are represented by $g(\sigma, \beta)$. Here, a single source views several detectors simultaneously and data are collected by different detectors corresponding to various angles, $\sigma$. This process is repeated for different values of $\beta$ to get the CT data, $g(\sigma, \beta)$.

The FBG mode can be implemented for a rotating object system also. The data collection mechanism remains the same as that for the PBG case.

\section{Tomographic inversion formula}

The process of retrieving (or reconstructing) a two-dimensional function from its projection data is similar for the FBG and PBG cases. The parallel beam CT formulation involves Fourier transforms while the fan-beam formulation incorporates Hilbert transforms in the inversion process.

\subsection{Parallel-beam formulation}

The inversion formula for the PBG case is based on the 'central-slice' theorem for Fourier transforms of two-dimensional functions. The theorem states that the one-dimensional Fourier transform of the projection data, $p(s ; \theta)$, with respect to the first variable $s$, is equal to the two-dimensional Fourier transform of the object function $f$ being imaged. Mathematically,

$$
\hat{f}(F ; \theta)=\hat{p}(F ; \theta) \text {. }
$$

Taking the inverse Fourier transform of (5) we get

$$
f(r, \phi)=\int_{0}^{\pi} \int_{-\infty}^{\infty} \hat{p}(F ; \theta) \exp [-i 2 \pi F r \cos (\theta-\phi)]|F| \mathrm{d} F \mathrm{~d} \theta .
$$

Here, for a given $\theta$,

$$
\hat{p}(F ; \theta)=\int_{-R}^{R} p(s ; \theta) \exp [-i 2 \pi F s] \mathrm{d} s .
$$


Equation (6) requires projection data on a continuous basis for all values of $s$ and $\theta$. For computational feasibility a finite cut-off has to be incorporated for the Fourier frequency. This cut-off is usually implemented in the form of a filter function, $W(F)$, which vanishes for $|F|$ greater than $A$, the cut-off frequency. Thus the filtered version of (6) becomes,

$$
\tilde{f}(r, \phi)=\int_{0}^{\pi} \int_{-\infty}^{\infty} \hat{p}(F ; \theta) \exp [-i 2 \pi F r \cos (\theta-\phi)] W(F)|F| \mathrm{d} F \mathrm{~d} \theta
$$

Here, the reconstruction, $\tilde{f}$, is approximate due to the finite cut-off introduced in the computations. Also, the discrete nature of the cT data forces the practical implementation of $A$ to be governed by the sampling theorem (Brigham 1974), i.e.,

$$
A \geqslant[1 /(2 \Delta s)] \text {. }
$$

The band-limiting filter, introduced in electron micrography by Ramachandran \& Lakshminarayanan (1970) is given by

$$
W(F)=\left(\begin{array}{ll}
1, & |F|<A, \\
0, & |F| \geqslant A,
\end{array}\right.
$$

and a popular 'sinc' filter used by Shepp \& Logan (1974) is given by,

$$
W(F)=\left(\begin{array}{cc}
{[\sin (\pi F / 2 A)] /(\pi F / 2 A),} & |F|<A, \\
0, & |F| \geqslant A .
\end{array}\right.
$$

\subsection{Fan-beam formulation}

The inversion formula for the FBG case was first derived by Herman \& Naparstek (1978) and is given by,

$$
f(r, \phi)=\left(1 / 4 \pi^{2}\right) \int_{0}^{2 \pi} \int_{-B}^{B}\left[1 / \sin \left(\sigma^{\prime}-\sigma\right)\right] D_{v} g(\sigma, \beta) \mathrm{d} \sigma \mathrm{d} \beta,
$$

where,

$$
\begin{aligned}
& g(\sigma, \beta)=\text { data for the ray represented by }(\sigma, \beta), \\
& D_{v} g(\sigma, \beta)=(1 / U)[(\partial g / \partial \sigma)-(\partial g / \partial \beta)], \\
& \sigma^{\prime}=\tan ^{-1}\{[r \cos (\beta-\phi)] /[D+r \sin (\beta-\phi)]\}, \\
& U=\left\{[r \cos (\beta-\phi)]^{2}+[D+r \sin (\beta-\phi)]^{2}\right\}^{1 / 2},
\end{aligned}
$$

and the other variables are as in the PBG case (see figure 2). We note that $U$ is the distance of the radiation source from $(r, \phi)$, the point being reconstructed, and $\sigma^{\prime}$ is the angular displacement of the particular data ray passing through that point $(r, \phi)$. A major difference [compared to (6)] is the computation of partial derivatives of the data, $g$.

\section{Convolution back-projection algorithm}

The reconstruction algorithms based on the inversion formulae summarised in $\S 3$, are known as 'transform methods'. It was observed by several researchers that these transform methods were slow and required very accurate interpolation schemes 
in computing the two-dimensional inverse Fourier transforms. The introduction of convolutions (Bracewell \& Riddle 1967; Ramachandran \& Lakshminarayanan 1970) eliminated the step of computing the Fourier transform of the data and the subsequent time consuming two-dimensional Fourier inversion.

\subsection{Parallel-beam algorithm}

Exploiting the convolution property of Fourier transforms, (8) can be rewritten as,

where,

$$
\tilde{f}(r, \phi)=\int_{0}^{\pi} \int_{-R}^{R} p(s ; \theta) q\left(s^{\prime}-s\right) \mathrm{d} s \mathrm{~d} \theta,
$$

and,

$$
q(s)=\int_{-A}^{A} W(F)|F| \exp [i 2 \pi F s] \mathrm{d} F,
$$

$$
s^{\prime}=r \cos (\theta-\phi) \text {. }
$$

Here, $q$ known as the convolving function and is the inverse Fourier transform of the filter function, $W(F)$. Equations (10)-(11) are the fundamental equations of the convolution back-projection (СBP) algorithm of CT. The inner integral of (10) is a convolution and the outer integral was termed back-projection by the early researchers. The CBP algorithm is the most widely used method of reconstruction employed by commercial CT scanners available for medical imaging purposes.

\subsection{Fan-beam algorithm}

The inversion formula given by (9) involves a singular integral thus forcing a regularization of the integral for computational purposes. A regularized version of (9) is given by (Herman \& Naparstek 1978)

where,

$$
f(r, \phi)=\frac{D}{4 \pi^{2}} \int_{0}^{2 \pi} \frac{1}{U^{2}} \int_{-R}^{R}\left(F_{1}+F_{2}\right) g(\sigma, \beta) \mathrm{d} \sigma \mathrm{d} \beta,
$$

$$
\begin{aligned}
& F_{1}=Q^{1}\left(\sigma^{\prime}-\sigma\right) \cos \sigma, \\
& F_{2}=Q^{2}\left(\sigma^{\prime}-\sigma\right) \cos \sigma^{\prime}, \\
& Q^{1}(u)=[-u Q(u)] / \cos ^{2}(u), \\
& Q^{2}(u)=[Q(u)+u(\mathrm{~d} Q / \mathrm{d} u)] / \sin (u), \\
& Q(u)=-2 \int_{0}^{A} W(F) \sin (2 \pi F u) \mathrm{d} F .
\end{aligned}
$$

A suitable choice of the filter function, $W(F)$, leads to the convolving functions $Q^{1}$ and $Q^{2}$. An implementatior of (12) for two-phase air-water bubbly flow in a pipe has been reported by Munshi et al (1987).

\subsection{Filter selection for the convolution algorithms}

A basic problem of the CBP algorithm of $\mathrm{Cr}$ is the choice of an appropriate filter, $W(F)$, for both the parallel-beam and fan-beam cases. Chang \& Herman (1980) have 
conducted an exhaustive study of several types of filters; other details are given by Herman (1980). Recently, a theoretical study was completed by Munshi (1989) to study the reconstruction quality of the CBP algorithm employing the popular filters reported in literature. Certain 'higher order' filters, based on the existing Fourier filters, have also been developed and some preliminary results involving simulated data have been reported by Arora et al (1988).

A new filter has been proposed by Rathore et al (1989) for the CBP algorithm and the simulated data results appear to be quite encouraging. Unlike the Fourier filters, this new filter is induced from the configuration, of the data collection geometry.

\section{Algebraic reconstruction methods}

The previous sections summarised the tomographic reconstruction formula for the PBG and the FBG modes of data collection which were classified as 'transform' methods. A whole group of iterative schemes have also been developed which reconstruct the function from its projection data. Such methods are known as 'algebraic' techniques.

These iterative algorithms start off with an assumed distribution, $f_{0}$, of the object function as the first step. Next, projection data are generated on the computer for $f_{0}$. The mismatch in the generated data and the actual data is computed and $f_{0}$ is corrected to get the next best approximation, $f_{1}$. A sequence of such approximations is shown (Tanabe 1971) to converge to the actual solution $f$, the distribution of the object function. This method was originally proposed by Kaczmarz (1937) for solving a system of linear equations.

Hounsfield (1973) employed this algebraic reconstruction technique (ART) to obtain images in the first CT scanner. At the same time Gordon et al (1970) also proposed ART independently for reconstructions. Later on, several variations of ART were also investigated; details are available elsewhere (Censor 1983).

All the ART-type methods (Censor 1983) have been reported to consume much more computer time than the transform methods (Lewitt 1983), in particular the CBP algorithm. This is the reason for medical scanners employing CBP although the first set of successful reconstructions was obtained using ART.

\section{Application in multi-phase flow studies}

The previous sections briefly outlined the theory and the implementation aspects of some tomographic techniques, the CBP algorithm in particular. Current two-phase flow research, and multi-phase flow studies in general, need an accurate measurement of void-fraction distribution across the flow cross-section. Tomographic reconstruction of void-fraction in air-water flow was studied by Munshi (1979) and related findings were reported by Schlosser et al (1980), DeVuono et al (1980) and Kulacki et al (1980). Improved reconstruction results were reported by Seshadri et al (1986).

The collection of $\mathrm{CT}$ data can be quite easy for the flow situation where the density distributions do not change rapidly with time as was the case in a previous study (Munshi 1979). For such situations a simple single source and single detector system suffices. The source can be a $\gamma$-ray source of at least milli-curie strength but a higher source is always beneficial in reducing the data collection time and/or reducing the statistical errors encountered in the counting of photons by the detector. The single 
source-detector system, mounted on a platform can be rotated to collect projection data in either the FBG mode or the PBG mode. Such a simple mechanism does not require rotation of the object and this is crucial for flow experiments as rotation of objects will generally be ruled out as is the case with the medical CT scanners where patients just cannot be rotated. However, if sufficient detectors are available then they can be arranged in a fan beam mode with a single $\gamma$-ray source to collect $\mathbf{C T}$ data at a much faster rate and also for flows which have void/density distributions changing with time. We note that the interval of counting (say $10 \mathrm{~s}$ ) will be the interval during which the flow distribution will still have to be assumed to be constant. This interval of counting can be reduced by using radiation sources of higher strength. $A$ simulation study has already been reported by DeVuono et al (1980).

The CT data thus obtained can be processed with any standard algorithm but the CBP method is recommended. The reconstructed function will be a distribution of the absorption coefficient, $\mu$, hence suitable calibration is required to convert the $\mathrm{Cr}$ numbers to the density values. For a two-phase gas-liquid flow, the mixture density, $\rho_{m}$, is related to the phase densities by the following relation,

$$
\rho_{m}=(1-\alpha) \rho_{l}+\alpha \rho_{g},
$$

If the gas-density is much smaller than the liquid density, then

$$
\rho_{m} \approx(1-\alpha) \rho_{l},
$$

and for steam-water flow we can write,

$$
\rho_{m}=1-\alpha \text {. }
$$

\section{New tomographic algorithms}

All the previous studies employing the CBP algorithm appear to be an overkill for the bubbly air-water flow situation where the projection data were roughly radially symmetric.

A series of tomographic methods were developed by Rathore et al (1986, 1987, 1988) for performing void-fraction reconstruction in radially symmetric situations generally occurring in pipe flows. A simplifiction of the CBP method leads to the Bessel function algorithm (Rathore et al 1987a, 1988a) incorporating the CBP filters. The algorithm involving radial polynomials (RAP) reported earlier (Rathore et al 1986, 1987b) results in a continuous approximation of the object function. The chordsegment inversion (CSI) technique (Rathore et al 1987c, 1988b) incorporates a discrete ring-type formulation.

All these three methods give a one-shot solution to the reconstruction process as data are required only from one 'view' for a radially symmetric situation. The CBP implementation requires convolution computations to be performed for all values of. $\theta$ as per (8). Thus computation time is considerably reduced if the new methods are used instead of the CBP method. For a 'rough' density distribution the CSI formulation appears to be the most suited while for a relatively 'smooth' distribution the RAP method is likely to give better results. The Bessel algorithm is a direct consequence of the CBP inversion technique for radial symmetry but it requires computation of the Fourier transform of the Cr data. A summary of these methods appears elsewhere (Munshi \& Rathore 1990). 
The distributions generally encountered in engineering situations are likely to be non-smooth and, hence, the CSI algorithm is recommended as a first-choice cr method. All the tomographic methods hold a lot of promise as diagnostic tools in studying the two-phase flow phenomenon. Multi-phase flow situations can also be handled with similar hardware and software facilities as the number of phases do not affect the CT method which regards density/void-fraction as a general function of position.

\section{Some sample reconstruction results}

This section outlines some of the results from a previous study (Rathore et al 1988c).

\subsection{Results with simulated data}

A general practice adopted in the studies was to test the algorithms by performing reconstructions with data generated by some known test functions. The test functions selected in most cases were of the following types: (i) constant; (ii) linear; (iii) exponential; (iv) step-function.
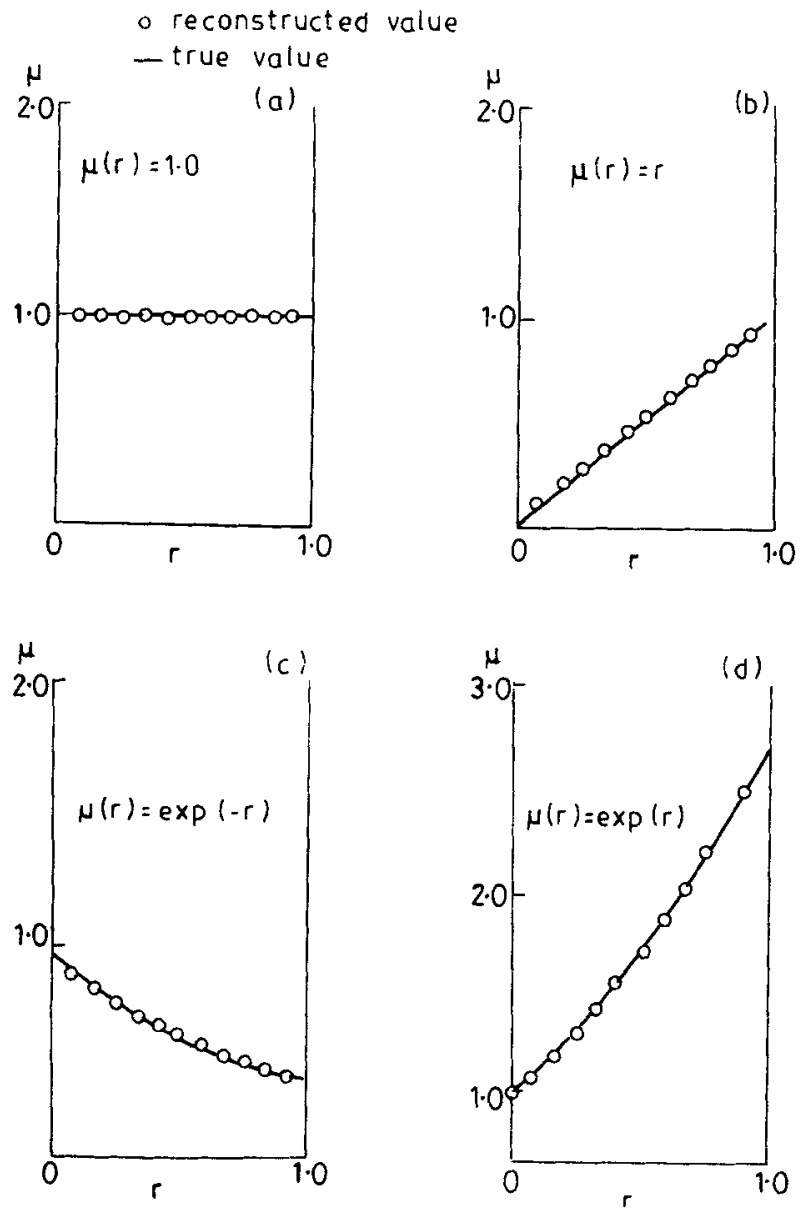

Figure 3. Reconstruction results for the (a) constant, (b) linear, (c) negative exponential and (d) positive exponential for a simulation of flow in a circular pipe of unit radius. 
These functions are reasonable simulations of the likely density/void distributions encountered in bubbly and annular two-phase flow situations. Figure 3 shows the reconstruction results for the constant, linear, and positive/negative exponentials for a simulation of flow in a circular pipe of unit radius. The excellent agreement in the reconstructed values and the original values is evident. Figure 4 depicts a similar reconstruction exercise for two simulations of annular flows. Again, the reconstructed values match well with the true values.

\subsection{Results for air-water flows}

The bubbly air-water flow data (Munshi 1979) was processed for several cases of cross-sectionally averaged values of density/void distribution and several sets of data were taken. Reconstructions for one such set, designated Scan 1, is shown in figure 5 . The density/void measurements are reported as 10 points in a pipe of $7.5 \mathrm{~cm}$ radius. In the experiment, $\mathrm{Cr}$ data were collected at 10 equally-spaced angular positions (intervals of $2.5^{\circ}$ ) in a fan-beam mode, hence the reconstruction is made available for 10 radial positions. In case a finer grid is required, data have to be collected for a finer angular-spacing. For better accuracy a stronger $\gamma$-ray source (greater than the 13.6 milli-curie one used in the study) is recommended.

\section{List of symbols}

$\begin{array}{ll}A & \text { Fourier cut-off frequency; } \\ B & \text { angle of extreme data-rays; } \\ c & \text { chord (path) of integration; } \\ D & \text { distance of source from origin; } \\ D_{v} & \text { partial derivative of } g(\sigma, \beta) ;\end{array}$

- reconstructed value

- actual value

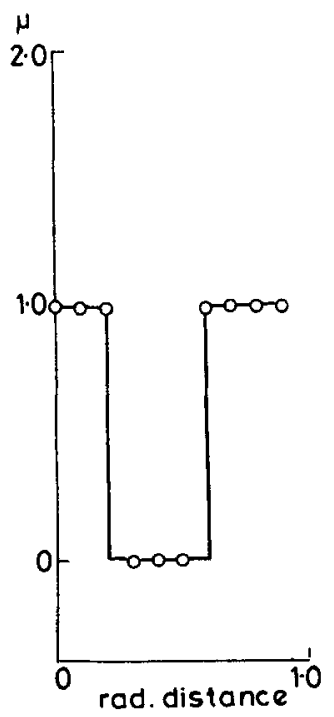

(a)

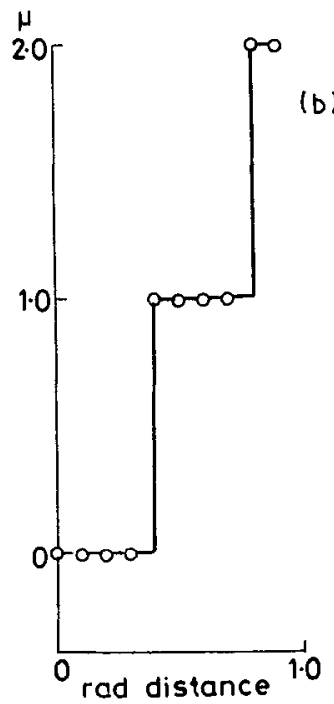

Figure 4. Reconstruction results for simulated annular flows. 


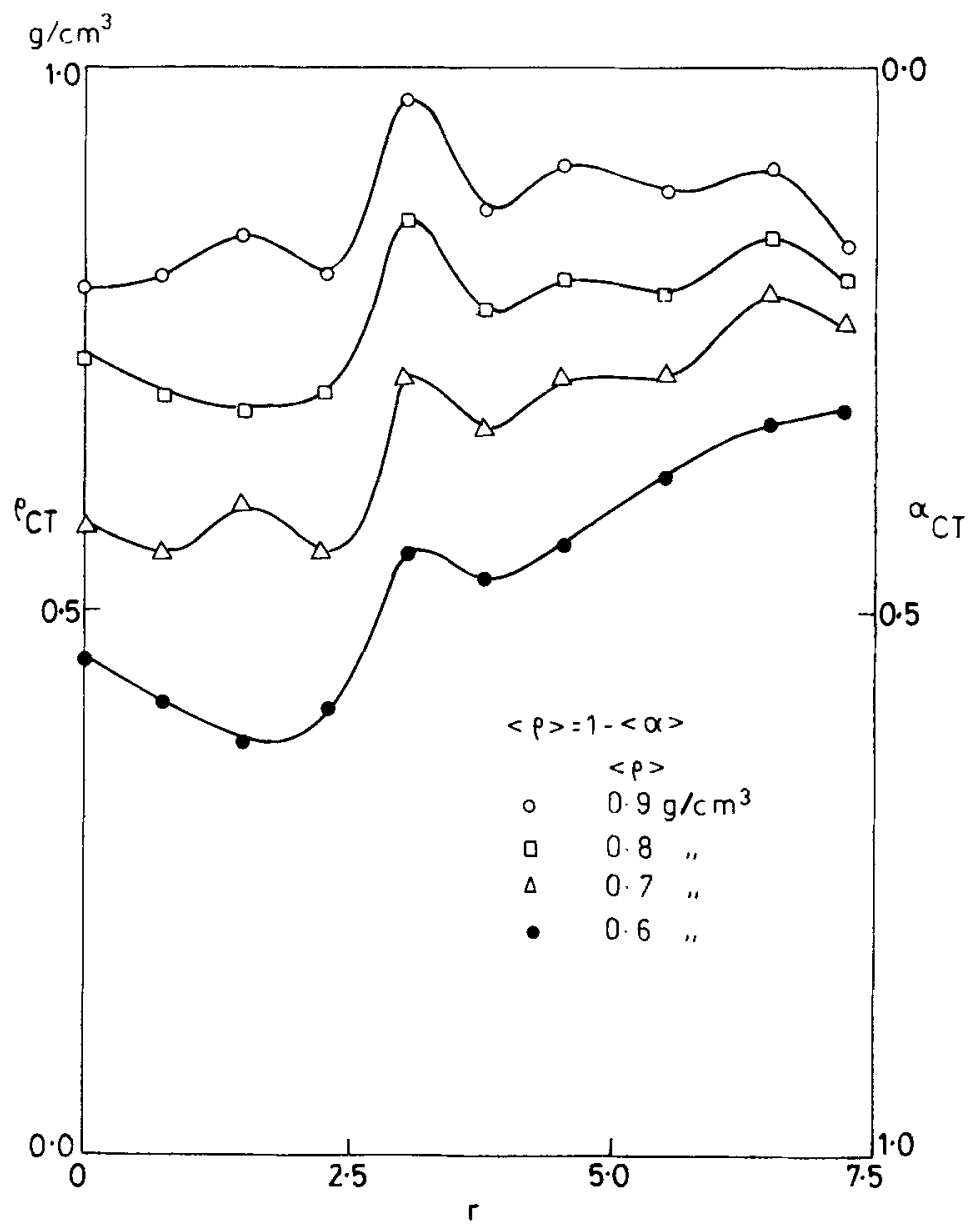

Figure 5. Reconstruction results for air-water flow for scan 1.

$f(r, \phi) \quad$ function being reconstructed;

$\tilde{f}(r, \phi) \quad$ reconstruction of $f(r, \phi)$;

$F, \hat{\Lambda} \quad$ Fourier frequency and Fourier transform, respectively;

$g(\sigma, \beta) \quad$ projection data (CT data) for fan beams;

$l \quad$ variable of integration $(\mathrm{cm})$;

$\Delta l \quad$ thickness of absorbing medium;

$N \quad$ radiation detector reading (counts $/ \mathrm{s}$ );

$N_{0} \quad$ radiation source strength (counts $/ \mathrm{s}$ );

$p(s ; \theta) \quad$ projection data (CT data) for parallel beams;

$r, \phi \quad$ cylindrical co-ordinates;

$R \quad$ radius of the object;

$s \quad$ perpendicular distance of data-ray from origin;

$s^{\prime} \quad s$-distance of data-ray passing through $(r, \phi)$;

$\Delta s \quad$ spacing between data-rays;

$U$ distance of point $(r, \phi)$ from the source;

$W(F) \quad$ window (filter) function;

$\alpha \quad$ void-fraction at point where $\rho_{m}$ is measured; 
$\beta \quad$ source position (fan beam geometry);

$\gamma \quad$ gamma (ray);

$\theta \quad$ source position (parallel beam geometry);

$\mu \quad$ absorption co-efficient $\left(\mathrm{cm}^{-1}\right)$;

$\rho \quad$ density $\left(\mathrm{g} / \mathrm{cm}^{3}\right)$;

$\rho_{l}, \rho_{g}, \rho_{m}$ density of liquid and gaseous phases, and of the mixture, respectively;

$\sigma^{\prime} \quad$ angle of data-ray passing through the point $(r, \phi)$;

$\langle$ cross-sectionally averaged quantity.

\section{References}

Arora P, Munshi P, Rathore R K S 1988 Higher order tomographic filters for non-destructive testing purposes. Nucl. Technol. 83: 228-230

Brigham E O 1974 The fast Fourier transform (Englewood Clifs, Nu: Prentice Hall)

Bracewell R N 1956 Strip integration in radio astronomy. Aust. J. Phys. 9: 198-217

Bracewell R N, Riddle A C 1967 Inversion of fan-beam scans in radio astronomy. Astrophys. J. 150: 427-434

Censor Y 1983 Finite series-expansion methods. Proc. IEEE 71: 409-419

Chang T, Herman G T 1980 A scientific study of filter selection for a fan-beam convolution algorithm. SIAM J. Appl. Math. 39: 83-105

Cormack A M 1963 Representation of a function by its line integrals, with some radiological applications. J. Appl. Phys. 34: 2722-2727

Deans S R 1983 The Radon transform and some of its applications (New York: John Wiley)

DeVuono A C, Schlosser P A, Kulacki F A, Munshi P 1980 Design of an isotopic Cr scanner for two-phase flow measurements. IEEE Trans. Nucl. Sci. NS-27: 814-820

Gordon R, Bender R, Herman G T 1970 Algebraic reconstruction technique (ART) for three-dimensional microscopy and X-ray photography. $J$. Theor. Biol. 29: 471-481

Herman G T 1980 Image reconstruction from projections: The fundamentals of computerized tomography (New York: Academic Press)

Herman G T, Naparstek A 1978 Fast image reconstruction based on a Radon inversion formula appropriate for rapidly collected data. SIAM J. Appl. Math. 33: 511-533

Hounsfield G N 1973 Computerized transverse axial scanning tomography. Part I: Description of the system. Br. J. Radiol. 46: 1016-1022

Kaczmarz M S 1937 Angenaherte auflosung von systemen linearer gleichungen. Bull. Acad. Polonaise Sci. Lett. Classe Sci. Math. Natur. Serier A35: 355-357

Kulacki F A, Schlosser P A, DeVuono A C, Munshi P 1980 A preliminary study of the application of reconstruction tomography to void-fraction measurements in two-phase flow. Proc. ANS/ASME/NRC Topical Meeting on Nuclear Reactor Thermal-Hydraulics NUREG/CP-0014, Saratoga Springs (New York) pp. 904-922

Lewitt R M 1983 Reconstruction algorithms: transform methods. Proc. IEEE 71: 390-408

McClellan G C, Tow D M 1986 Neutron tomography of damaged nuclear fuel bundles. Neutron Radiography, Proc. Second World Conference. (Paris: D Reidel) pp. 711-718

Miyoshi S, Tanimoto Y, Uyuma K, Sano K 1987 The evaluation of scC defects of steel piping using high-energy X-ray ct Scanner. Nucl. Eng. Design 102: 275-287

Munshi $P 1979$ Two-phase flow studies in the bubbly-flow regime using a scanning gamma-ray densitometer, M S Thesis, Ohio State University, Columbus, Ohio

Munshi P 1989 Error estimates for the convolution back-projection algorithm in computerized tomography, Ph D Thesis, Indian Institute of Technology, Kanpur

Munshi P, Rathore R K S 1990 Some new tomographic methods for multi-phase flow situations. Advances in mechanical engineering (ed.) R S Agarwal (New Delhi: Tata McGraw Hill) pp. 717-725

Munshi P, Rathore R K S, Swamy S T, Dhariyal I D 1987 Tomographic reconstruction of the density distribution using direct fan-beam algorithms. Nucl. Instrum. Methods Phys. Res. A257: 398-405

Natterer F 1986 The mathematics of computerized tomography (New York: John Wiley \& Sons)

Radon J 1917 Uber die bestimmung von funktionen durch ihre integralwarte langs gewisser mannigfaltigkeiten. Berichte Sachsische Akademie der Wissenschaften Leipzig. Math. - Phys. Kl 69: 262-267 
Ramachandran G N, Lakshminarayanan A V 1970 Three dimensional reconstruction from radiographs and electron micrographs: application of convolution instead of Fourier transforms. Proc. Natl. Sci. Acad. USA 68: 2236-2240

Rathore R K S, Dhariyal I D, Munshi P, Seshadri M D 1986 Tomographic reconstruction using radial polynomiais. Trans. Am. Nucl. Soc. 52: 407-409

Rathore R K S, Munshi P, Arora P, Malik S D, Vaish A K, Singh K S, Singh U 1989 A new non-Fourier tomographic filter for image reconstruction. Nucl. Technol. 85: 346-349

Rathore R K S, Munshi P, Bhatia V K, Pandimani S 1987a Point-density measurements in radially symmetric flows using Bessel functions. Trans. Am. Nucl. Soc. 54: 176-178

Rathore R K S, Munshi P, Bhatia V K, Pandimani S 1988a Filtered Bessel functions in computerized tomography. Nucl. Eng. Design 108: 375-384

Rathore R K S, Munshi P, Dhariyal I D, Swamy S T 1987b Tomographic reconstruction of the density field using radial polynomials. $\mathrm{Nucl}$. Technol. 78: 7-12

Rathore R K S, Munshi P, Jarwal R K 1987c Measuremeñt of void-fraction distribution using a tomographic chord-segment inversion technique. Am. Soc. Mech. Engrs. FED 50: 164-166

Rathore R K S, Munshi P, Jarwal R K, Dhariyal I D 1988b Investigation of the bubbly air-water flow using the chord-segment inversion algorithm. Nucl. Technol. 82: 227-234

Schlosser P A, DeVuono A C, Kulacki F A, Munshi P 1980a Analysis of high-speed CT scanners for non-medical applications. IEEE Trans. Nucl. Sci. NS-27: 780-794

Schlosser P A, DeVuono A C, Kulacki F A, Munshi P 1980b Cross-sectional density measurements of multi-phase flow using computerized tomography, Argonne National Laboratory, $A N L-80-62$

Seshadri M D, Munshi P, Dhariyal I D, Rathore R K S 1986 Application of digital tomography in two-phase flow studies. Nucl. Instrum. Methods Phys. Res A251: 577-582

Shepp L A, Logan B F 1974 The Fourier reconstruction of a head section. IEEE Trans. Nucl. Sci. NS-21: $21-43$

Tanabe K 1971 Projection method for solving a singular system. Numer. Math. 17: 203-214 